\title{
两种异质结太阳能电池聚合物供体材料的设计与理论性质
}

\author{
刘小锐陈春香何荣幸吕伟* 李明* \\ (西南大学化学化工学院 重庆 400715)
}

\begin{abstract}
摘要 模拟了以苯并噻吩作为富电子基团分别与 $1 H$-benzo $[d][1,2,3]$ triazole 和 $1 H$-benzo $[d][1,2,3]$ triazole- 6 -carbonitrile 作 为缺电子基团构成的两种聚合物太阳能电池供体材料(PBnDT-HTAZ, PBnDT-6CNTAZ)及 PC60BM 为受体材料的理论 性质. 利用 DFT 理论分析了两种聚合物的电子和光物理性质, 通过 Marcus 理论研究了供-受体化合物在供受体界面的 电荷转移性质和供体聚合物的空穴迁移能力. 计算结果表明: 供体聚合物具有强而宽的吸收, 并且具有强的分子内电 子转移和从电子供体到电子受体的分子间电子转移, 对应的复合物都具有较小的激子束缚能; 与 PBnDT-HTAZ 相比, 设计的供体 PBnDT-6CNTAZ, 由于引入了强吸电性的氧基而具有更大的开路电压和更好的抗氧化能力, 另外, 在供受 体界面具有更好的电荷转移特性, 并且在供体中具有相对大的空穴迁移速率. 因此, 可以推断得知引入氰基的 PBnDT-6CNTAZ 是一种潜在的更好的太阳能电池供体材料.
\end{abstract}

关键词 聚合物太阳能电池; 供-受体化合物; 苯并三唑; 电子转移

\section{Theory Design of Two Polymer Donors for Organic Heterojunction Solar Cells}

\author{
Liu, Xiaorui Chen, Chunxiang He, Rongxin Shen, Wei* Li, Ming* \\ (School of Chemistry and Chemical Engineering, Southwest University, Chongqing 400715)
}

\begin{abstract}
A highly efficient organic solar cell needs a copolymer as donor possessing low-band gap, high open-circuit voltage $\left(V_{\text {oc }}\right)$, good charge transfer at donor-acceptor (D-A) interface, and good transport properties in polymers. As the first step towards this goal, we constructed two polymers (PBnDT-HTAZ and PBnDT-6CNTAZ) incorporating benzodithiophene $(\mathrm{BnDT})$ as the electron-rich units and either $1 H$-benzo $[d][1,2,3]$ triazole (HTAZ) or $1 H$-benzo $[d][1,2,3]$ triazole-6-carbonitrile (6CNTAZ) as the electron-deficient unit. The designed PBnDT-6CNTAZ was introduced a cyano in the electron-deficient unit. PBnDT-HTAZ and PBnDT-6CNTAZ as donors and [6,6]-phenyl C61-butyric acid methyl ester (PC60BM) as an acceptor in hetero-organic solar cell, as well as the D-A complexes are investigated using density functional theory (DFT) and time-dependent density functional theory (TD-DFT) in this work. The electronic properties, optical absorption properties, intramolecular and intermolecular charge transfer, exciton binding energy of two polymers and corresponding D-A complexes are discussed. By means of Marcus theory, the exciton-dissociation, charge-recombination rate of D-A complex at the D-A interface, and the hole-transport rate of polymer donors are studied. According to the computational results, it indicates that the two polymers have strong and wide absorption peaks in visible region, as well as strong intramolecular charge transfer and intermolecular charge transfer for corresponding D-A complex. The designed PBnDT-6CNTAZ possesses lower the energy of the highest occupied molecular orbital (HOMO) so that PBnDT-6CNTAZ exhibits larger open-circuit voltage and better ability of antioxidant in comparison with PBnDT-HTAZ. The two polymers corresponding D-A complexes have small exciton binding energy. The PBnDT-6CNTAZ has good ability of charge transfer and larger hole-transport rate in comparison to PBnDT-HTAZ. It is thus clear that incorporating cyano in polymer (PBnDT-6CNTAZ) have an important impact on performance of organic solar cell. Therefore, we can infer that PBnDT-6CNTAZ may be a potential donor material of bulk heterojunction solar cells. The present calculated results demonstrated a first attempt of providing a theoretical model for the introduction of electron-deficient groups in copolymer donors. These results may provide a structural guideline for optimizing chemical construction of copolymer donors to improve the performance of bulk heterojunction solar cells. Our computational models and conclusions can be applied to design and predict other new polymer donors.

Keywords organic solar cell; donor-acceptor complex; benzotriazole; charge transfer
\end{abstract}

\section{1 引言}

由于聚合物太阳能电池具有成本低、易制造、重量
轻等特点 ${ }^{[1]}$, 因而受到广泛的关注并得到快速的发展. 其中活化层是异质结太阳能电池的关键部分, 由电子供 体和电子受体共混制备而成 ${ }^{[2,3]}$, 富勒烯及其衍生物是

*E-mail: shenw@swu.edu.cn, liming@swu.edu.cn

Received September 7, 2012; published October 17, 2012.

Supporting information for this article is available free of charge via the Internet at http://sioc-journal.cn

Project supported by the National Natural Science Foundation of China (No. 21073144), the Natural Science Foundation Project of CQ CSTC (No. CSTC, 2009BB4104), and the Fundamental Research Funds for the Central Universities (No. XDJK2010B009).

项目受国家自然科学基金(No. 21073144)、重庆市自然科学基金(No. CSTC, 2009BB4104)、中央高校基本科研业务费(No. XDJK2010B009)资助. 
典型的电子受体 ${ }^{[3,4]}$. 有机太阳能电池是一个光电转化 的设备, 其能量转化过程分为三个基本步骤 ${ }^{[5]}$ : (1)光激 发活化层分子, 产生激子; (2)激子扩散到电子给体(D) 和电子受体(A)的异质结界面处发生电荷分离, 生成电 子和空穴载流子; (3)电荷传输到电极而产生电流. 研究 表明, 供体材料的能隙在 $1.9 \mathrm{eV}$ 左右有利于太阳光的吸 收 ${ }^{[3,6]}$, 另外, 激子和电荷在传输过程中的损失要尽可能 小.

由于咔唑及其类似物具有较大的电子亲和势, 因而 常作为吸电子基团被广泛应用于 D-A 模型的聚合物 ${ }^{[7]}$. 据文献报道, 含有咔唑的聚合物太阳能电池效率超过 $7.0 \%{ }^{[8]}$. 最近, You 等 ${ }^{[9]}$ 报道了以苯并三唑 $(1 H$-benzo$[d][1,2,3]$ triazole)作为吸电子基团的高效 D-A 聚合物太 阳能电池材料(PBnDT-HTAZ), 其光谱响应范围从 350 到 $650 \mathrm{~nm}$. 聚合物供体 PBnDT-HTAZ 是由苯并噻吩作 为供电子基团和苯并三唑作为吸电子基构成的，它与 PC60BM 受体共混形成的异质结太阳能电池的效率达 到 $4.36 \%$.

基于 PBnDT-HTAZ 的分子结构, 为了了解在聚合 物中引入强吸电基团以后对聚合物供体的光物理性质 等参数的影响并与 PBnDT-HTAZ 作比较, 我们在吸电 子基团 $1 H$-benzo[d][1,2,3]triazole 上引入了一个强吸电 性的氧基, 并研究了这两种聚合物作为异质结太阳能电 池中的供体材料的理论性质.

\section{2 方法}

所有计算均在 Gaussian09 程序下 ${ }^{[10]}$, 在曙光 HPC 高性能集群计算机上完成. 供体聚合物、受体 PC60BM 和供体-受体化合物的基态结构采用密度泛函理论 (DFT), 在 B3LYP/6-31G(d)方法和基组下优化 ${ }^{[11]}$, 光学 参数是在与基态结构优化相同的方法和基组上采用含 时密度泛函(TD-DFT) 计算得到 ${ }^{[12]}$, 电荷转移密度可以 由 Multiwfn 直观显示 ${ }^{[13]}$. 在 Marcus ${ }^{[14]}$ 理论中涉及到的 交换积分、重组能等参数均是在 B3LYP/6-31G(d)方法和 基组水平上计算的. 供体一受体化合物电子转移中的交 换积分还用到了 Generalized Mulliken-Hush (GMH)模 型 ${ }^{[15]}$ 和有限场方法 ${ }^{[3]}$.

\section{3 结果与讨论}

\section{1 结构和电子性质}

两种供体聚合物及受体 PC60BM 的化学结构如图 1 所示. 两种聚合物是由苯并塞吩 $(\mathrm{BnDT})$ 作为供电子基 团分别与苯并三唑 $(1 H$-benzo[d][1,2,3]triazole, 简写为 HTAZ)和引入氧基的苯并三唑(1H-benzo $[d][1,2,3]-$ triazole-6-carbonitrile, 简写为 6CNTAZ)作为吸电子基 团组成. 对应供体聚合物的名称缩写分别为 PBnDT-HTAZ 和 PBnDT-6CNTAZ, 其中, PBnDT-
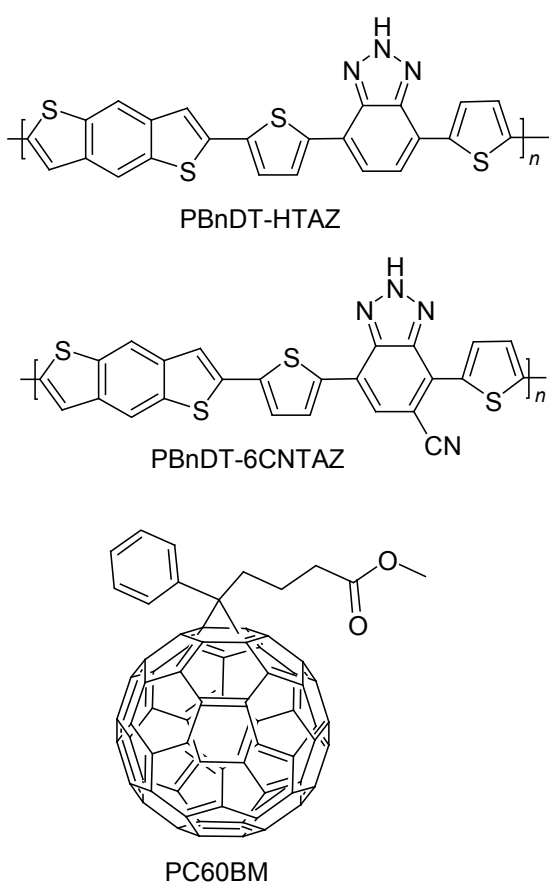

图 1 供体聚合物和受体 PC60BM 分子结构图

Figure 1 Sketch map of the structure of polymer donors and PC60BM acceptor

HTAZ 已经被合成并应用于聚合物太阳能电池 ${ }^{[9]}$, PBnDT$6 \mathrm{CNTAZ}$ 是设计的一种新聚合物. PC60BM 作为电子受 体. 当供体和受体结合在一起, 简称供-受体化合物, 如: (PBnDT-HTAZ) $)_{2}$-PC60BM 和(PBnDT-6CNTAZ) $)_{2}$-PC60BM. 我们分别分析了供体聚合物、受体 PC60BM 以及供-受 体化合物的基态性质和开路电压.

供体聚合物的前线轨道能级、能隙与太阳能电池的 开路电压、光学性质以及电荷的分离直接相关 ${ }^{[6]}$. 聚合 物的能级、能隙以及 $\mathrm{H}_{\mathrm{D}}-\mathrm{L}_{\mathrm{A}}$ 见表 1 . 相对低的 HOMO 能 级有更强的抗氧化能力 ${ }^{[6(a)]}$, 另外, 开路电压与供体 HOMO 与受体 LUMO 的能级差 $\left(\mathrm{H}_{\mathrm{D}}-\mathrm{L}_{\mathrm{A}}\right)$ 成正比, 通常在 理论上可以通过下列方程式来估算开路电压 ${ }^{[16]}$ :

$$
V_{\mathrm{oc}}=(1 / e)\left(\left|E_{\mathrm{HOMO}}(\mathrm{D})\right|-\left|E_{\mathrm{LUMO}}(\mathrm{A})\right|\right)-0.3 \mathrm{~V}
$$

其中, $e$ 表示元电子, $E_{\mathrm{HOMO}}(\mathrm{D})$ 和 $E_{\mathrm{LUMO}}(\mathrm{A})$ 分别表示供体 的 HOMO 能量和受体的 LUMO 能量, 0.3 为经验常数, 因此, 相对大的 $\mathrm{H}_{\mathrm{D}}-\mathrm{L}_{\mathrm{A}}$ 具有相对大的开路电压. 从表中 数据明显看出, 不管是单体还是二聚物, PBnDT-

表 1 一聚、二聚物的轨道能级能量 $(\mathrm{eV})$ 以及供体 $\mathrm{HOMO}$ 与受体 LUMO 的差值

Table 1 Orbital energy levels of monomers and dimers and the values of $\mathrm{H}_{\mathrm{D}}-\mathrm{L}_{\mathrm{A}}$

\begin{tabular}{cccccccccc}
\hline & \multicolumn{3}{c}{$n=2$} & & \multicolumn{3}{c}{$n=1$} \\
\cline { 2 - 3 } \cline { 7 - 9 } & HOMO LUMO & $E_{\mathrm{g}}$ & $\mathrm{H}_{\mathrm{D}}-\mathrm{L}_{\mathrm{A}}$ & & HOMO & LUMO & $E_{\mathrm{g}}$ \\
\hline PBnDT-HTAZ & -4.83 & -2.50 & 2.33 & 1.81 & & -5.00 & -2.28 & 2.72 \\
PBnDT-6CNTAZ & -5.10 & -2.89 & 2.21 & 2.08 & & -5.27 & -2.66 & 2.61 \\
\hline
\end{tabular}


6CNTAZ 的 HOMO 能级均低于 PBnDT-HTAZ, 而且 PBnDT-6CNTAZ 具有相对小的能隙, PBnDT-6CNTAZ 的 $\mathrm{H}_{\mathrm{D}}-\mathrm{L}_{\mathrm{A}}$ 大于 PBnDT-HTAZ. 因此, PBnDT-6CNTAZ 具 有相对强的抗氧化能力和较高的开路电压.

为了得到更多关于化学结构与电子性质的关系, 我们对聚合物做了态密度分析(图 2). 态密度可以用于 判断不同基团对每个分子轨道的贡献 ${ }^{[3]}$. 二聚物 $(\mathrm{PBnDT}-\mathrm{HTAZ})_{2}$ 的态密度见图 2(a), 对部分已占据轨 道, -8.0 和 $-7.0 \mathrm{eV}$ 能量附近主要是 HTAZ 和桥环的 贡献, 在 $-5.8 \mathrm{eV}$ 附近 PBnDT 的贡献较大, 从图中可以 看出两个片段对 HOMO 都有一定贡献, 而 LUMO 的贡 献主要是来源于 HTAZ 及噻吩桥环, 分析表明, 聚合物 PBnDT-HTAZ 中 HTAZ 和桥环噻吩部分更容易接受电 子. 从图 2(b) 总态密度和投影态密度图可以看出 $(\mathrm{PBnDT}-6 \mathrm{CNTAZ})_{2}$ 与 $(\mathrm{PBnDT}-\mathrm{HTAZ})_{2}$ 具有类似的性质.

受体 PC60BM 的 $\mathrm{HOMO}=-5.66 \mathrm{eV}, \mathrm{LUMO}=$ $-3.02 \mathrm{eV}$, 能隙 $E_{\mathrm{g}}=2.64 \mathrm{eV}$. 之所以选用 PC60BM 作 为电子受体, 是因为它具有较大的电子亲和能(较低的 LUMO 能级).

众所周知, 有机太阳能电池活化层是由电子受体和 电子供体构成. 于是, 我们通过态密度来分析供-受体 化合物中各个基团对轨道的贡献有助于进一步了解供 受体之间电子供受的关系. 图 2(c)表示化合物
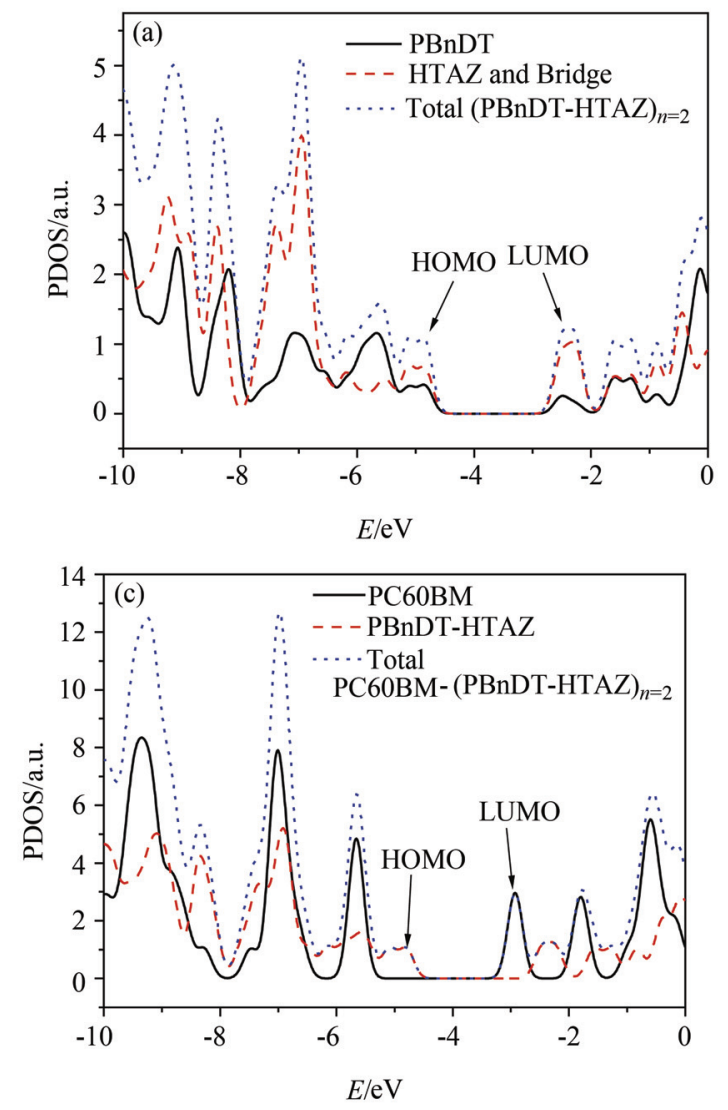

PC60BM-(PBnDT-HTAZ) 2 的态密度, 从图中可以看出, 对 HOMO 轨道的贡献几乎都是来源于供体 PBnDTHTAZ, 对 LUMO 轨道是由受体 PC60BM 贡献的. 化合 物 PC60BM-(PBnDT-6CNTAZ) 2 态密度见图 2(d), 同样, $\mathrm{HOMO}$ 轨道主要是来源于电子供体 PBnDT-6CNTAZ 的 贡献，而 LUMO 主要是来源于 PC60BM 的贡献. 通过对 化合物总态密度和投影态密度的分析很明显可以看出, 当化合物受到光激发，聚合物 (PBnDT-HTAZ, PBnDT-6CNTAZ)更容易给出电子，PC60BM 更容易接 受电子.

\section{2 光学性质以及电子转移}

光学吸收是有机太阳能电池设备的一个重要的性 质参数, 电子供体要具有宽而强的吸收范围. 以下分别 对供体聚合物和供-受体化合物的光学吸收以及它们在 吸收中的电子转移作了讨论. 模拟的供体吸收光谱列于 图 3 中.

聚合物的光学吸收参数和光学吸收中轨道的贡献 都列于表 2 中. 由表 2 数据可得，随着聚合度的增加，最 大吸收波长红移，聚合物 PBnDT-6CNTAZ 最大吸收波 长相对于 PBnDT-HTAZ 明显红移. 从图 3 可以看出, 在 近紫外和可见光区域有 4 个相对较强的吸收. 电子转移 可以通过差分密度图直观地看出(图 4). PBnDT-HTAZ, 吸收较强的三个激发态是 $\mathrm{S}_{1}, \mathrm{~S}_{4}, \mathrm{~S}_{11}, \mathrm{~S}_{1}$ 态主要是
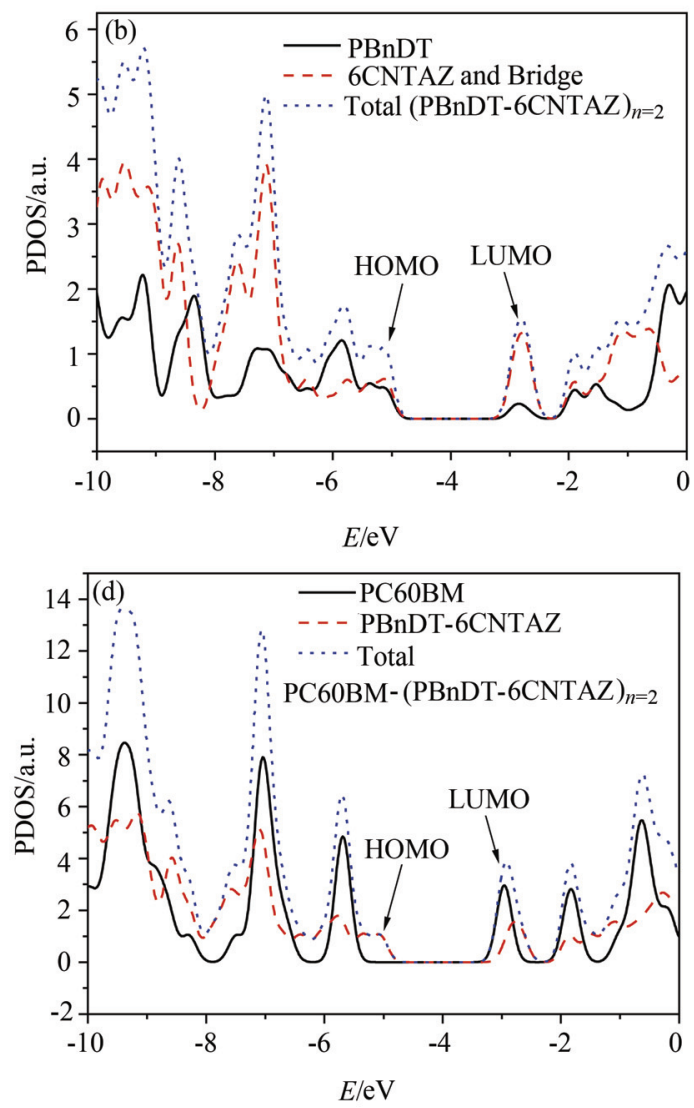

图 2 聚合物供体、供体一受体化合物的总态密度和投影态密度图

Figure 2 Total densities of states (TDOS) and projected DOS (PDOS) of all polymers and D-A complexes 
表 2 一聚、二聚物光学吸收参数和二聚物光学吸收的轨道贡献

Table 2 Calculated absorption wavelength $(\lambda)$, oscillator strength $(f)$, and main configuration of monomers and dimers

\begin{tabular}{|c|c|c|c|c|c|c|}
\hline & \multicolumn{4}{|r|}{$n=2$} & \multicolumn{2}{|c|}{$n=1$} \\
\hline & & $\lambda / \mathrm{nm}$ & $f$ & Major configuration & $\lambda / \mathrm{nm}$ & $f$ \\
\hline \multirow{5}{*}{ PBnDT-HTAZ } & $\mathrm{S}_{1}$ & 563 & 3.51 & $\mathrm{H} \rightarrow \mathrm{L}(94 \%)$ & \multirow[t]{5}{*}{468} & 1.42 \\
\hline & $\mathrm{S}_{4}$ & 422 & 0.43 & $\mathrm{H}-1 \rightarrow \mathrm{L}+1(82 \%)$ & & \\
\hline & \multirow{3}{*}{\multicolumn{2}{|c|}{$\mathrm{S}_{11} 356$}} & & $\mathrm{H}-3 \rightarrow \mathrm{L}+1(17 \%)$ & & \\
\hline & & & 0.16 & $\mathrm{H}-1 \rightarrow \mathrm{L}+2(13 \%)$ & & \\
\hline & & & & $\mathrm{H} \rightarrow \mathrm{L}+3(36 \%)$ & & \\
\hline \multirow{5}{*}{ PBnDT-6CNTAZ } & $\mathrm{S}_{1}$ & 579 & 2.94 & $\mathrm{H} \rightarrow \mathrm{L}(97 \%)$ & \multirow[t]{5}{*}{524} & 0.98 \\
\hline & $\mathrm{S}_{4}$ & 444 & 0.26 & $\mathrm{H}-1 \rightarrow \mathrm{L}+1(80 \%)$ & & \\
\hline & \multirow{3}{*}{\multicolumn{2}{|c|}{$\mathrm{S}_{12} 352$}} & & $\mathrm{H}-4 \rightarrow \mathrm{L}+1(33 \%)$ & & \\
\hline & & & 0.27 & $\mathrm{H}-1 \rightarrow \mathrm{L}+2(10 \%)$ & & \\
\hline & & & & $\mathrm{H} \rightarrow \mathrm{L}+3(35 \%)$ & & \\
\hline
\end{tabular}

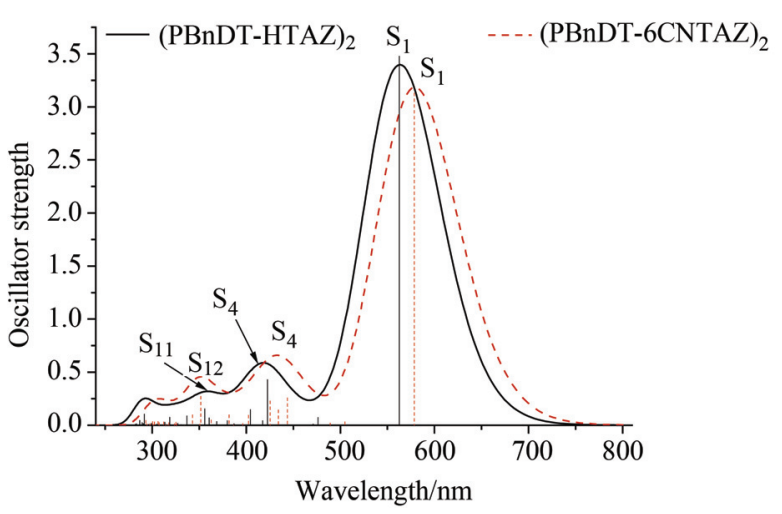

图 3 聚合物的吸收光谱模拟图

Figure 3 Simulated absorption spectra of polymers

$\mathrm{HOMO} \rightarrow \mathrm{LUMO}$ 的跃迁, 从转移密度图 4 得出, 分子内 最低电子转移态 $\mathrm{S}_{1}$ 的电子转移主要是从整个分子链到 HTAZ; $\mathrm{S}_{4}$ 态与 $\mathrm{S}_{1}$ 类似; $\mathrm{S}_{11}$ 态的电子转移是整个分子本 身之间的转移. PBnDT-6CNTAZ, 吸收较强的有 $\mathrm{S}_{1}, \mathrm{~S}_{4}$, $\mathrm{S}_{12}$, 对应的电子转移性质与 PBnDT-HTAZ 的类似. 从 上文总态密度和投影态密度图分析结果显示, 聚合物供 体在受到光激发下 6CNTAZ 和 HTAZ 部分更容易接受 电子, 从电荷差分密度得知, 两种聚合物都表现出由 $\mathrm{HOMO} \rightarrow \mathrm{LUMO}$ 轨道跃迁发生的电子转移是从整个分 子链到吸电子基.

供一受体化合物对应的激发态轨道贡献和电荷差分 密度见图 5. 对化合物 (PBnDT-HTAZ) $)_{2}$-PC60BM, 吸收 最强的 $\mathrm{S}_{10}$ 态, 主要是由 $\mathrm{HOMO} \rightarrow \mathrm{LUMO}+3$ 轨道跃迁, 从电荷差分密度图可以看出, 其电子密度是定域在电子 供体 PBnDT-HTAZ 上的, 电子转移的方向是从 PBnDT-HTAZ 的两端向中间转移, 而 $\mathrm{S}_{1}$ 态主要是由 $\mathrm{HOMO} \rightarrow \mathrm{LUMO}$ 轨道的跃迁, 空穴几乎都定域在供体 PBnDT-HTAZ 上, 电子集中于受体 PC60BM 上. 从图 5 可以推断出, 分子间电子转移的方向是从供体

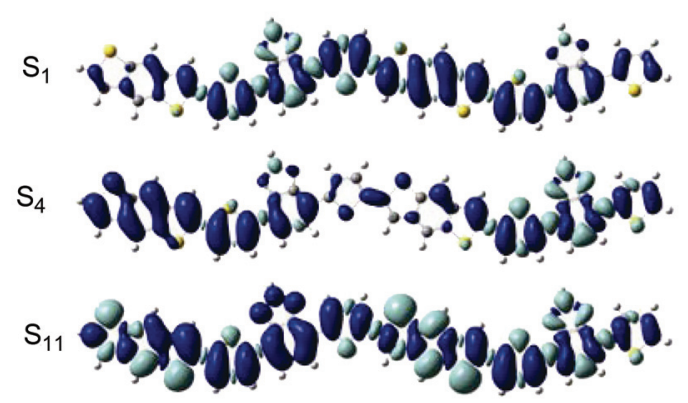

PBnDT-HTAZ

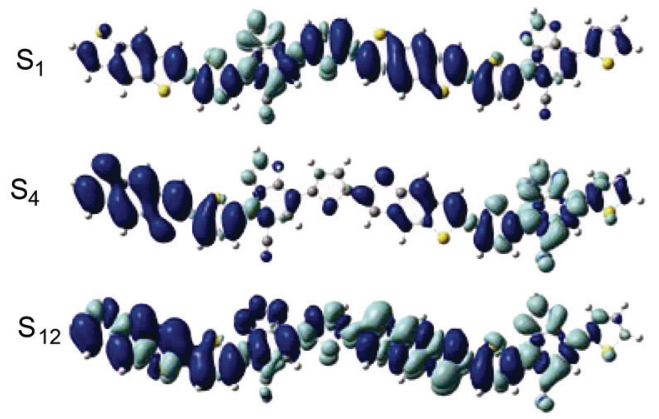

PBnDT-6CNTAZ

图 4 聚合物供体的电荷差分密度图(蓝色和白色区域分别表示空穴 和电子)

Figure 4 Charge difference densities plots of the polymers (the blue area and the white area stand for the hole and electron, respectively)

$\mathrm{S}_{1}:$

$H \rightarrow L(99 \%)$

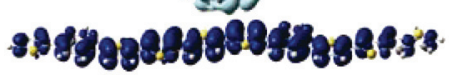

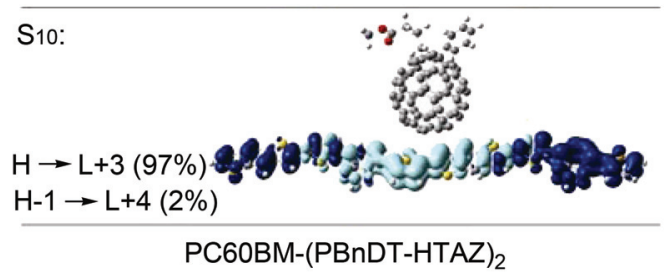

$\mathrm{S}_{1}$ :

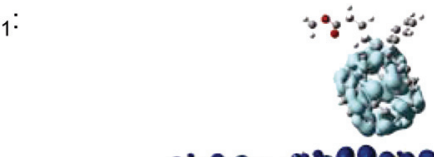

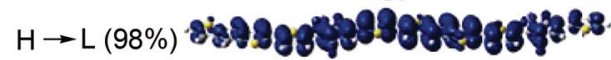

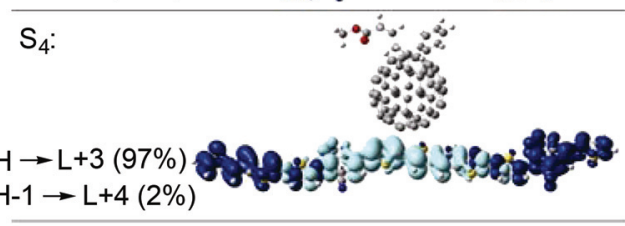

PC60BM-(PBnDT-6CNTAZ) 2

图 5 供-受体化合物电荷密度差分图以及激发态轨道贡献

Figure 5 Charge difference densities plots of donor-acceptor complexes 
PBnDT-HTAZ 到受体 PC60BM, $\mathrm{S}_{1}$ 态是化合物(PBnDT$\mathrm{HTAZ})_{2}-\mathrm{PC} 60 \mathrm{BM}$ 的最低分子间电荷转移态; 从图 5 很 明显看出，化合物 (PBnDT-6CNTAZ) $)_{2}$ PC60BM 与 PC60BM-(PBnDT-HTAZ) 2 具有类似的光学性质和电子 转移性质, 其中吸收最强的是 $\mathrm{S}_{4}$ 态.

\section{3 激子扩散与激子束缚能}

激子扩散到供受体界面发生分裂, 定域在供体 LUMO 轨道的电子转移到受体的 LUMO 轨道形成电荷 转移态. 通常, 在供受体界面处激子分离有两步, 首先 是分离为具有较强束缚力的极化子对, 然后分离为自由 的极化子 ${ }^{[3,17]}$. 激子束缚能主要起源于电子和空穴之间 的库伦相互作用 ${ }^{[18]}$. 为了激子有效地分离为极化子, 需 要克服大的激子束缚能. 对光电器件来说, 激子束缚能 是一个很关键的参数. 在有机太阳能电池中, 激子束缚 能与电荷分离直接相关 ${ }^{[3]}$. 在理论上通常用下列方程式 来估算 ${ }^{[17,18]}$ :

$$
E_{\text {coul }}=\mathrm{IP}-\mathrm{EA}-E_{\mathrm{opt}}
$$

其中, IP, EA 和 $E_{\mathrm{opt}}$ 分别表示供受体复合物的电离势, 电 子亲和能以及光学能隙(第一单重激发能), 第一单重激 发能是用 TDDFT 在相同基态结构计算水平之上得到 ${ }^{[18]}$. 有机太阳能电池的激子束缚能在 $0.2 \sim 1 \mathrm{eV}$ 范围内 ${ }^{[17]}$, (PBnDT-6CNTAZ)-PC60BM 和(PBnDT-HTAZ)$\mathrm{PC} 60 \mathrm{BM}$ 复合物的激子束缚能分别为 0.264 和 $0.262 \mathrm{eV}$.

\section{4 激子在供受体界面的分离与电荷重组速率}

激子在界面处的分离和重组速率通常用 Marcus 理 论来估算，其表达式如下 ${ }^{[3,14]}$ :

$$
k=\frac{\left|V_{\mathrm{DA}}\right|^{2}}{\hbar^{2}} \sqrt{\frac{\pi}{\lambda k_{\mathrm{B}} T}} \exp \left(\frac{-\left(\Delta G+\lambda^{2}\right)}{4 \lambda k_{\mathrm{B}} T}\right)
$$

其中, $k$ 表示速率; $\lambda$ 表示重组能; $V_{\mathrm{DA}}$ 表示初始态和终态 间的电子耦合矩阵元(电荷转移积分); $\Delta G$ 表示电子转移 反应的自由能变化; $k_{\mathrm{B}}$ 玻尔兹曼常数; $h$ 表示普朗克常 数; $T$ 是温度 $(T=300 \mathrm{~K})$, 激子分离和电荷重组过程自由 能的改变分别表示为 $\Delta G_{\mathrm{CT}}$ 和 $\Delta G_{\mathrm{CR}}$. 算 $[15,19]$.

电荷转移积分可以通过两态的 $\mathrm{GMH}$ 模型来估

$$
V_{\mathrm{DA}}=\frac{\mu_{\mathrm{TR}} \Delta E}{\sqrt{(\Delta \mu)^{2}+4\left(\mu_{\mathrm{TR}}\right)^{2}}}
$$

其中, 两态间的跃迁偶极距 $\mu_{\mathrm{TR}}$ 的方向是从电子供体到 电子受体(见图 $1 \mathrm{~S}$ 沿着 $Y$ 轴方向, 支持信息); $\Delta \mu$ 表示初
态和末态的偶极距之差; $\Delta E$ 是垂直激发能. 方程(4)中 $\Delta \mu$ 由有限场差分方法得到.

重组能包括内重组能和外重组能，供受体复合物最 低电荷转移激发态衰退至基态的内重组能表达式如

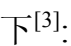

$$
\lambda=E\left(\mathrm{~A}^{-}\right)-E(\mathrm{~A})+E(\mathrm{D})-E\left(\mathrm{D}^{+}\right)
$$

其中, $E\left(\mathrm{~A}^{-}\right)$表示在阴离子结构上电中性受体的能量; $E(\mathrm{~A})$ 优化的电子受体基态能量; $E(\mathrm{D})$ 表示在电中性结构 上电子供体阳离子的能量; $E\left(\mathrm{D}^{+}\right)$是优化的供体阳离子 的能量. 两种分子的内重组能都约为 $0.19 \mathrm{eV}$, 与 PC60BM：PDDTT 供受体化合物的内重组能相近(0.194 $\mathrm{eV})^{[3]}$. 外重组能是分子在溶剂中由整体的几何弛豫引 起电子极化而产生的, 但是在理论上很难估算供受体化 合物在固态下的外重组能. 目前, 在实验上对总重组能 的研究结果显示, corrole-fullerene 化合物在极性溶剂中 总的重组能约为 $0.75 \mathrm{eV}^{[20]}$, 于是, 在本工作中总重组 能设为 $0.75 \mathrm{eV}$. 电荷重组反应的自由能变化 $\Delta G_{\mathrm{CR}}$ 等于 供体的电离势和受体的电子亲和势之差 ${ }^{[3]}$. 激子分离反 应的自由能变化 $\Delta G_{\mathrm{CT}}$ 由 Rehm-Weller 方程得到 ${ }^{[21]}$ :

$$
\Delta G_{\mathrm{CT}}=\mathrm{EA}(\mathrm{A})-\mathrm{IP}(\mathrm{D})-\Delta E_{0-0}-E_{\text {coul }}
$$

式(6)中, $\Delta E_{0-0}$ 和 $E_{\mathrm{coul}}$ 分别为供体的最低单重态激发能 和供受体复合物的电荷转移态的库伦作用能.

作为异质结太阳能电池活化层的供受体材料, 当激 子分离时, 电荷转移速率越大, 电荷重组速率越小, 其 对设备的效率贡献越大. 两种复合物在相同堆积方式下 (见图 $1 \mathrm{~S}$, 支持信息)计算的 $V_{\mathrm{DA}}, \lambda, \Delta G$, 激子分离速率 $\left(k_{\mathrm{CT}}\right)$ 和电荷重组速率 $\left(k_{\mathrm{CR}}\right)$ 列于表 3 . 从表中数据可以得 出, 与 PBnDT-HTAZ 相比较, 设计的聚合物 PBnDT-6CNTAZ 具有更强的电子耦合强度, 而内重组 能相差不大. PBnDT-HTAZ 电荷转移速率比 PBnDT6CNTAZ 高出一个数量级, 然而, PBnDT-6CNTAZ 电荷 重组速率比 PBnDT-HTAZ 小 3 个数量级. 因此, 我们认 为设计的 PBnDT-6CNTAZ 作为电子供体具有相对好的 电荷转移能力.

\section{5 供体聚合物的空穴迁移速率}

当激子在供受体界面处发生电荷分离以后，形成的 电子和空穴紧接着向两极传输 ${ }^{[22]}$. 若温度足够高, 根据 Marcus 理论, 供体材料的空穴迁移速率 $\left(k_{\mathrm{h}}\right)$ 为 $^{[14,23]}$.

$$
K_{\mathrm{h}}=\left(t_{\mathrm{h}}^{2} / \hbar^{2}\right) \sqrt{\pi / \lambda_{\mathrm{h}} k_{\mathrm{B}} T} \exp \left(-\lambda_{\mathrm{h}}^{2} / 4 k_{\mathrm{B}} T\right)
$$

在式(7)中, 决定传输速率主要有两个参数：电子交换积

表 3 供受体化合物的转移积分 $(\mathrm{eV})$, 内重组能 $(\mathrm{eV})$, 吉布斯自由能 $(\mathrm{eV})$ 以及电荷分离和重组速率

Table 3 Computed charge transfer integral $V_{\mathrm{DA}}(\mathrm{eV})$, inner reorganization energy $\lambda(\mathrm{eV})$, free enthalpy of the reaction $\Delta G$ (eV), the rates of exciton-dissociation $k_{\mathrm{CT}}$, and rate of charge-recombination $k_{\mathrm{CR}}$ of two D-A complexes.

\begin{tabular}{lllllll}
\hline & $V_{\mathrm{DA}}$ & $\lambda_{\text {in }}$ & $\Delta G_{\mathrm{CR}}$ & $\Delta G_{\mathrm{CT}}$ & $k_{\mathrm{CT}} / \mathrm{s}^{-1}$ & $k_{\mathrm{CR}} / \mathrm{s}^{-1}$ \\
\hline PBnDT-HTAZ & 0.0545 & 0.1978 & -1.8123 & -0.6287 & $4.74 \times 10^{13}$ & $2.75 \times 10^{7}$ \\
PBnDT-6CNTAZ & 0.1083 & 0.1947 & -2.0784 & -0.1268 & $1.51 \times 10^{12}$ & $2.98 \times 10^{4}$ \\
\hline
\end{tabular}


分 $t$ 和重组能 $\lambda$. 根据 Koopman 定理, 对于阳离子体系 的电子转移反应(即空穴转移), 电子耦合强度是相邻两 个片段中性体系 $\mathrm{HOMO}$ 与 $\mathrm{HOMO}-1$ 的能量差的一 半 ${ }^{[23,24]}$.

$$
t_{\mathrm{h}}=\frac{E_{\mathrm{HOMO}}-E_{\mathrm{HOMO}-1}}{2}
$$

重组能 $\lambda_{\mathrm{h}}=\lambda_{1}+\lambda_{2}\left(\lambda_{1}\right.$ 是离子态下中性结构与阳离 子结构的能量之差, $\lambda_{2}$ 是中性态下中性结构与阳离子结 构的能量之差 $)^{[25]}$.

作为异质结太阳能电池活化层的供体材料要具有 良好的空穴传输能力, 供体材料的空穴传输速率越大越 有利于提高空穴载流子的迁移率. 两种聚合物的二聚体 片段在相同的面对面堆积方式下(见图 2S, 支持信息), 表 4 中列出了供体聚合物的空穴转移交换积分 $t_{\mathrm{h}}$, 空穴 重组能 $\lambda_{\mathrm{h}}$ 以及空穴传输速率 $k_{\mathrm{h}}$. 从表中数据可以看出, PBnDT-6CNTAZ 具有相对大的空穴转移交换积分和相 对小的重组能, 从而使 PBnDT-6CNTAZ 具有相对大的 空穴传输速率。因此, 我们推测设计的供体 PBnDT-6CNTAZ, 相比于 PBnDT-HTAZ 而言, 具有更 好的空穴迁移能力.

表 4 聚合物供体的交换积分 $(\mathrm{eV})$, 重组能 $(\mathrm{eV})$ 以及空穴迁移速率 Table 4 Computed hole transfer integral $(\mathrm{eV})$, hole reorganization energy $(\mathrm{eV})$, and transport rates of hole for polymer donors

\begin{tabular}{llll}
\hline & $t$ & $\lambda$ & $k_{\mathrm{h}} / \mathrm{s}^{-1}$ \\
\hline PBnDT-HTAZ & 0.02190 & 0.1484 & $4.96 \times 10^{12}$ \\
PBnDT-6CNTAZ & 0.02231 & 0.1422 & $5.58 \times 10^{12}$ \\
\hline
\end{tabular}

\section{4 结论}

本文计算模拟了异质结太阳能电池中的两种供体 聚合物(PBnDT-HTAZ 和 PBnDT-6CNTAZ) 以及供-受体 化合物的理论特征. 利用 DFT 理论分析了两种聚合物 的基态性质和光物理性质, 并通过 Marcus 理论比较研 究了两种供-受体复合物在供受体界面处的激子分离、 电荷重组速率和供体的空穴传输能力. 结果表明: (1)设 计的供体 PBnDT-6CNTAZ 具有强而宽的吸收范围, 相 比于 PBnDT-HTAZ, 其最大波长红移; (2)两种聚合物供 体都表现出从整个分子到吸电子单元强的分子内电子 转移, 分子间电子转移是从电子供体到电子受体的过 程, 两者都具有较小的激子束缚能; (3)与 PBnDT-HTAZ 相比较, PBnDT-6CNTAZ 具有更大的开路电压和更好 的抗氧化性; (4) PBnDT-6CNTAZ 电荷重组速率比 PBnDT-HTAZ 小 3 个数量级, 而且在聚合物中空穴传输 速率比 PBnDT-HTAZ大. 因此, 我们推测认为引入氭基 的 PBnDT-6CNTAZ 是一种潜在的更好的太阳能电池供 体材料.

\section{References}

[1] (a) Cho, S.; Lee, J.; Tong, M. H.; Seo, J. H.; Yang, C. Adv. Funct.
Mater. 2011, 21, 1910; (b) Chen, L.; Shen, X.; Chen, Y. Chin. J. Chem. 2012, 30, 2219; (c) Loser, S.; Bruns, C. J.; Miyauchi, H.; Ortiz, R. P.; Facchetti, A.; Stupp, S. I.; Marks, T. J. J. Am. Chem. Soc. 2011, 133, 8142; (d) Zhuo, Z.-L.; Zhang, F.-J.; Xu, X.-W.; Wang, J.; Lu, L.-F.; Xu, Z. Acta Phys.-Chim. Sin. 2011, 27, 875. (卓 祖亮, 张福俊, 许晓伟, 王健, 卢丽芳, 徐征, 物理化学学报, 2011, 27, 875.); (e) Ye, H.; Li, W.; Li, W. Chin. J. Org. Chem. 2012, 32, 266. (叶怀英, 李文, 李维实, 有机化学, 2012, 32, 266.)

[2] Zhang, J.; Deng, D.; He, C.; He, Y.; Zhang, M.; Zhang, Z.-G.; Zhang, Z.; Li, Y. Chem. Mater. 2011, 23, 817.

[3] Li, Y.; Pullerits, T.; Zhao, M.; Sun, M. J. Phys. Chem. C 2011, 115, 21865.

[4] (a) Walker, B.; Kim, C.; Nguyen, T.-Q. Chem. Mater. 2011, 23, 470; (b) Pappenfus, T. M.; Schmidt, J. A.; Koehn, R. E.; Alia, J. D. Macromolecules 2011, 44, 2354; (c) Zhang, T.; Piao, L.; Zhao, S.; Xu, Z.; Yang, L.; Liu, X.; Ju, S. Chin. J. Org. Chem. 2011, 31, 260. (张 天慧, 朴玲钜, 赵谡玲, 徐征, 杨否, 刘祥志, 鞠思婷, 有机化学, 2011, 31, 260.)

[5] (a) Yi, Y.; Coropceanu, V.; Brédas, J.-L. J. Mater. Chem. 2011, 21, 1479; (b) Chen, Z. X.; Huang, Y. C.; Li, Z.; Kang, G. J. Prog. Chem. 2009, 21, 2271. (陈兆旭, 黄玉成, 李哲, 康国俊, 化学进展, 2009, 21, 2271.)

[6] (a) Blouin, N.; Michaud, A.; Gendron, D.; Wakim, S.; Blair, E.; Neagu-Plesu, R.; Belletête, M.; Durocher, G.; Tao, Y.; Leclerc, M. J. Am. Chem. Soc. 2008, 130, 732; (b) Mikroyannidis, J. A.; Sharma, S. S.; Vijay, Y. K.; Sharma, G. D. Appl. Mater. Interfaces 2010, 2(1), 270 .

[7] (a) Tang, S. S.; Zhang, J. P. J. Phys. Chem. A 2011, 115, 5184; (b) Shang, H.; Fan, H.; Shi, Q.; Li, S.; Li, Y.; Zhan, X. Solar Energy Materials \& Solar Cells 2010, 94, 457; (c) Beaupré, S.; Belletête, M.; Durocher, G.; Leclerc, M. Macromol. Theory Simul. 2011, 20, 13.

[8] Shang, H.; Fan, H.; Liu, Y.; Hu, W.; Li, Y.; Zhan, X. Adv. Mater. 2011, 23, 1554

[9] Price, S. C.; Stuart, A. C.; Yang, L.; Zhou, H.; You, W. J. Am. Chem. Soc. 2011, 133, 4625.

[10] Frisch, M. J.; Trucks, G. W.; Schlegel, H. B.; Scuseria, G. E.; Robb, M. A.; Cheeseman, J. R.; Scalmani, G.; Barone, V.; Mennucci, B.; Petersson, G. A.; Nakatsuji, H.; Caricato, M.; Li, X.; Hratchian, H. P.; Izmaylov, A. F.; Bloino, J.; Zheng, G.; Sonnenberg, J. L.; Hada, M.; Ehara, M.; Toyota, K.; Fukuda, R.; Hasegawa, J.; Ishida, M.; Nakajima, T.; Honda, Y.; Kitao, O.; Nakai, H.; Vreven, T.; Montgomery, Jr., J. A.; Peralta, J. E.; Ogliaro, F.; Bearpark, M.; Heyd, J. J.; Brothers, E.; Kudin, K. N.; Staroverov, V. N.; Kobayashi, R.; Normand, J.; Raghavachari, K.; Rendell, A.; Burant, J. C.; Iyengar, S. S.; Tomasi, J.; Cossi, M.; Rega, N.; Millam, N. J.; Klene,M.; Knox, J. E.; Cross, J. B.; Bakken, V.; Adamo, C.; Jaramillo, J.; Gomperts, R.; Stratmann, R. E.; Yazyev, O.; Austin, A. J.; Cammi, R.; Pomelli, C.; Ochterski, J. W.; Martin, R. L.; Morokuma, K.; Zakrzewski, V. G.; Voth, G. A.; Salvador, P.; Dannenberg, J. J.; Dapprich, S.; Daniels, A. D.; Farkas, €. O.; Foresman, J. B.; Ortiz, J. V.; Cioslowski, J.; Fox, D. J. Gaussian 09, Revision A.01, Gaussian, Inc., Wallingford, CT, 2009.

[11] (a) Becke, A. D. J. Chem. Phys. 1993, 98, 5648; (b) Lee, C.; Yang, W.; Parr, R. G. Phys. Rev. B 1988, 37, 785; (c) Cohen, A. J.; Mori-Sánchez, P.; Yang, W. T. Chem. Rev. 2012, 112, 289.

[12] (a) Perdew, J. P.; Burke, K.; Ernzerhof, M. Phys. Rev. Lett. 1997, 78, 1396; (b) Sun, L.; Bai, F.-Q.; Zhao, Z.-X.; Zhang, H.-X. Solar Energy Materials \& Solar Cells 2011, 95, 1800.

[13] Lu, T. Multiwfn, version 2.1, http://multiwfn.codeplex.com/

[14] (a) Marcus, R. A. Rev. Mod. Phys. 1993, 65, 599; (b) Marcus, R. A. Ann. Rev. Phys. Chem. 1964, 15, 155.

[15] (a) Cave, R. J.; Newton, M. D. J. Chem. Phys. 1997, 106, 9213; (b) Cave, R. J.; Newton, M. D. Chem. Phys. Lett. 1996, 249, 15.

[16] (a) Bijleveld, J. C.; Verstrijden, R. A. M.; Wienk, M. M.; Janssen, R. A. J. Appl. Phys. Lett. 2010, 97, 073304 (1-3); (b) Scharber, M. C.; Mühlbacher, D.; Koppe, M.; Denk, P.; Waldauf, C.; Heeger, A. J.; Brabec, C. J. Adv. Mater. 2006, 18(6), 789.

[17] Rand, B. P.; Genoe, J.; Heremans, P.; Poortmans, J. Prog. Photovolt: Res. Appl. 2007, 15, 659.

[18] Zhen, C.-G.; Becker, U.; Kieffer, J. J. Phys. Chem. A 2009, 113, 9707.

[19] Vooren, A. V.; Lemaur, V.; Ye, A.; Beljonne, D.; Cornil, J. Chem. Phys. Chem. 2007, 8, 1240.

[20] D’Souza, F.; Chitta, R.; Ohkubo, K.; Tasior, M.; Subbaiyan, N. K.; 
Zandler, M. E.; Rogacki, M. K.; Gryko, D. T.; Fukuzumi, S. J. Am. Chem. Soc. 2008, 130, 14263.

[21] Shen, F.; Peng, A.; Chen, Y.; Dong, Y.; Jiang, Z.; Wang, Y.; Fu, H.; Yao, J. J. Phys. Chem. A 2008, 112, 2206.

[22] Shang, Y.; Li, Q.; Meng, L.; Wang, D.; Shuai, Z. Theor. Chem. Acc. 2011, 129, 291.

[23] Deng, W.-Q.; Goddard III, W. A. J. Phys. Chem. B 2004, 108, 8614.
[24] Lan, Y.-K.; Huang, C.-I. J. Phys. Chem. B 2008, 112, 14857.

[25] (a) Han, L. Z.; Wang, Z.; Hua, Y. J.; Ren, A. M.; Liu, Y. L.; Liu, P. J. Acta Chim. Sinica 2012, 70, 579. (韩立志, 王卓, 华英杰, 任爱 民, 刘䎦玲, 刘朋军, 化学学报, 2012, 70, 579.); (b) Ren, X.-F.; Ren, A.-M.; Feng, J.-K.; Sun, C.-C. J. Photochem. Photobiol. A: Chem. 2009, 203, 92 